\title{
Effects of Combined Functional Electrical Stimulation and Joint Mobilization on Muscle Activation and Mobility of Ankle Joints and Modified Functional Reach Test in Stroke Patients
}

\author{
Su-Jin Kim, PT $\cdot$ Ho-Hee Son ${ }^{1 \dagger}$
}

Department of Physical Therapy, Graduate School of Health Sciences, Catholic University of Pusan 'Department of Physical Therapy, College of Health Sciences, Catholic University of Pusan

Received: February 20, 2019 / Revised: February 22, 2019 / Accepted: March 18, 2019

(c) 2019 J Korean Soc Phys Med

\section{| Abstract |}

PURPOSE: This study was conducted to investigate the effects of combined Joint Mobilization and Functional Electrical Stimulation on Muscle Activation and Mobility of ankle joints in stroke patients and their Modified Functional Reach Test (MFRT) results.

METHODS: A total of 26 patients with stroke were randomly selected for enrollment in this study. (1) Functional Electrical Stimulation (FES) (2) combined Joint Mobilization and FES. An EMG system was used to measure tibialis anterior and gastrocnemius activities. Range Of Motion (ROM) of Ankle Joint and MFRT for Dynamic Balance. Pre and post intervention results were compared by paired-t-tests and differences in changes after intervention between groups were identified by the independent $t$-test.

RESULTS: The muscle activation, ROM, and MFRT differed significantly in the experimental group $(\mathrm{p}<.05)$. The

†Corresponding Author : Ho-Hee Son sonhh@cup.ac.kr, http://orcid.org/0000-0003-0905-6484

This is an Open Access article distributed under the terms of the Creative Commons Attribution Non-Commercial License (http://creativecommons.org/licenses/by-nc/3.0) which permits unrestricted non-commercial use, distribution, and reproduction in any medium, provided the original work is properly cited.
ROM was significantly different for the active dorsiflexion pre and post intervention in the group that received FES alone $(\mathrm{p}<.05)$.

CONCLUSION: The results of this study suggest use of a systematic program of proactive posture control to prevent dysfunction when planning interventions for ankle joints can help stroke patients walk efficiently.

Key Words: Ankle Mobilization, Functional Electrical Stimulation, Modified Functional Reach Test, Muscle Activation of Ankle

\section{Introduction}

Stroke patients suffer from damage to the central nervous system that causes spasticity and muscle weakness in the upper and lower extremities on the opposite side of the damage, resulting in difficulty in attaining normal gait. In particular, dorsiflexion does not occur on the ankle in the swing phase of gait and initial contact is impossible because of a foot drop in the stance phase [1]. In addition, the stiffness of the ankle joint leads to increased spasticity [2]; thus, treatment of the ankle joints is very important in the rehabilitation of stroke patients.

Functional electrical stimulation (FES) is often used in 
clinical practice to correct foot drop. Many studies have shown that FES is effective at inducing muscle contraction, thereby increasing the Range of Motion (ROM) [3], as well as enhancing muscular strength [4]. Moreover, studies of FES therapy have shown that it improves motor recovery of the lower limb in stroke patients and their gait ability when applied to the muscle quadriceps, hamstring, tibialis anterior, and medial gastrocnemius muscle [5]. Furthermore, application of FES to the peroneal nerve and tibialis anterior of stroke patients enhances muscle strength of the dorsiflexor and improves the spasticity of the plantar flexor and motor function [6]. Finally, technological development of FES has improved foot drop in stroke patients via recovery of gait ability and motor function in the rehabilitation of stroke patients [7].

In the rehabilitation of stroke patients, there is a need to improve passive and active movements by reinforcing muscle strength around the ankle as well as via electrical stimulation therapy [8]. Various joint mobilization methods have been conducted to treat contractures of ankle joints in stroke patients who suffer from progression of contractures for a long time because of spasticity.

When weight-bearing training was applied to a stroke patient along with joint mobilization, their ROM and walking speed were improved [9]. In addition, the application of posterior talofibular glide improved mobility of the ankle, muscle strength and balance ability [10]. When posterior talofibular glide was applied in combination with dorsiflexion, balance and gait function improved [11].

In particular, ankle joints are closely related to the balance ability in a sitting position, which plays a role in regulating delicate shaking of the body and is thus related to recovery of balance control [12]. In clinical practice, the Functional Reach Test (FRT) is widely used for balance evaluation in stroke patients. In some studies, FRT has been used in relation to the function of ankle joints. For example, ankle joint strategy exercise was used to evaluate the balance control ability of stroke patients [13] and the effects of ankle joint strategy training on the balance ability of stroke patients in the presence or absence of visual feedback has been investigated [14].

As shown above, many studies have applied FES and joint mobilization for functional improvement of ankle joints in stroke patients. However, there are insufficient studies on the application of FES and ankle joint mobilization in combination. Therefore, this study was conducted to investigate the effects of combined joint mobilization and FES on muscle activation and mobility of the ankle joint in stroke patients and their MFRT.

\section{Methods}

\section{Subjects}

This study investigated patients who were diagnosed with stroke and hospitalized at E - hospital located in Ulsan, South Korea for about two months from November 10, 2017 to January 11, 2018. Before the study, a cooperation letter was distributed to each ward and the primary care physicians. Overall, 26 patients undergoing central nervous system development therapy, functional rehabilitation training, and functional electrical stimulation therapy who understood the purpose of the study and agreed to participate voluntarily were included in this investigation. Inclusion criteria were as follows: diagnosed with stroke for more than three months, no orthopedic surgical experience or orthopedic disease, walking disturbance due to foot drop, able to communicate and understand this study. Exclusion criteria were: bilateral paralysis due to recurrent stroke and cerebral infarction, likely to develop peripheral neuropathy. This study was approved by the Institutional Review Board of the Catholic University of Busan (Approval number: CUPIRB-2017-033) prior to the start of the study. 


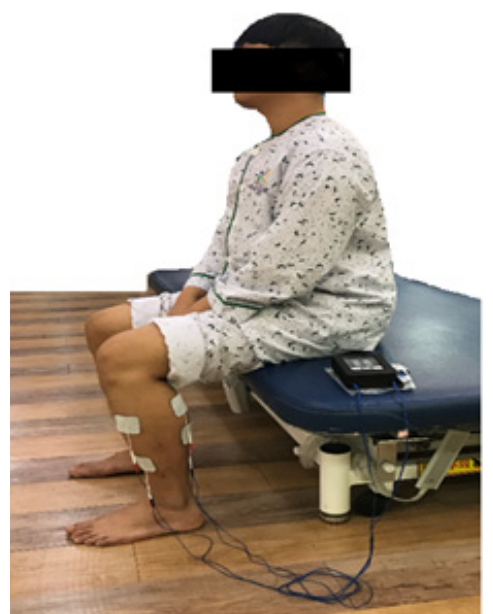

Fig. 1. Application of functional electrical stimulation

\section{Intervention}

\section{1) Functional Electrical Stimulation}

In this study, Microstim (Microstim, Medel Gmbk, Germany) was used as the FES therapy device. After receiving a full explanation of the purpose and method of the study, the subjects were divided into two groups, one that received FES only in the sitting position in a wheelchair and a another that received FES following joint mobilization. The functional electrical stimulation was set as Rectangular-Biphasic with $40 \mathrm{~Hz}$ frequency and $40 \mu \mathrm{s}$ amplitude [15]. Antagonist stimulation was used to facilitate the dorsiflexors induced ankle dorsiflexion and inhibition of plantaflexion spasticity during the stance phase [16]. To promote dorsiflexion, a pair of FES electrodes stimulated the proximal portion of the tibialis anterior (5 $\mathrm{cm}$ below the head of the fibula) [17] and the other pair of FES electrodes stimulated the valley of the lateral gastrocnemius [18] to promote plantar flexion. Both groups received sessions five times a week for 15 minutes per session (Fig. 1).

\section{2) Joint mobilization}

Ten physical therapists with more than five years of

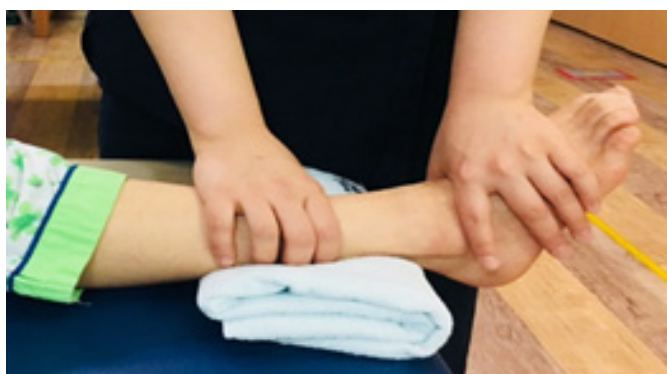

Fig. 2. Intervention of ankle joint mobilization (dorsiflexion)

experience participated in the ankle joint mobilization intervention.

To promote dorsiflexion, the patient put their leg on the treatment mat in a supine position with the heel protruding from the end of the mat. The therapist fixed the leg with their left hand and wrapped the foot with their fingers and thumb to keep the ankle in a stable position, then applied a grade I pulling force toward the tail side. At this time, the moving force pushed the tibia toward the talus bone, which caused the talus bone to glide backward (Fig. 2)

To promote plantar flexion, the patient put their leg on the treatment mat in a prone position with their foot protruding from the end of the mat as above. The therapist put their hand on the far side of the mortise at the back of the talus and the calcaneus and the moving force pushed the calcaneus forward with respect to the tibia. This action caused the talus to glide forward [19]. This method was applied to the experimental group five times a week for 15 minutes each (Fig. 3).

\section{Measurement}

\section{1) Muscle Activation}

LXM3204 (LAXTHA, Korea) was used to measure the EMG signals of the muscles. To compare the effects pre and post intervention, the electrodes were attached to the muscles around the ankle joints (tibialis anterior and lateral side of the gastrocnemius) $[20,21]$. The ground electrode 


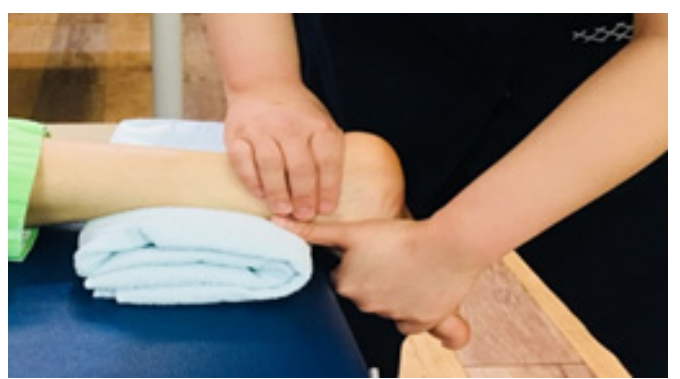

Fig. 3. Intervention of ankle joint mobilization (plantarflexion)

was attached to a close region so as not to interfere with the movement [22]. Before the electrodes were attached, the subjects were shaved with a razor and exfoliated with alcohol to reduce skin resistance. The sampling rate for collecting EMG signals was set to $1,024 \mathrm{~Hz}$ with a bandpass filter of $10-120 \mathrm{~Hz}$.

The raw data of the surface EMG signal were standardized by processing with the Root Mean Square (RMS). The reference voluntary contractions (RVC) value obtained for three seconds out of five seconds excluding the first and last seconds of the five seconds were used, and the average value of the three measurements was used. The RVC values for the tibialis anterior and gastrocnemius muscles were calculated while the subjects performed sitto-stand activity. For the trial, the EMG signal was collected while the subjects performed plantarflexion and dorsiflexion of the ankle joint. Data for each trial were expressed as a percentage of the calculated mean RMS of the percentage of a reference voluntary contraction ( $\% \mathrm{RVC})$, and the mean $\%$ RVC of three trials was used for analysis [23].

\section{2) Range of Motion; ROM}

The range of motion was measured using a Goniometer (PATTERSON, China). The normal ranges of dorsiflexion and plantar flexion of the ankle were $10^{\circ}-20^{\circ}$ and $40^{\circ}-50^{\circ}$, respectively, and measurements were made by the same person. The subject stretched their knees and placed their heels on the mat in a supine position, which was set as a basic position, after which the range was measured [20].

\section{3) Modified Functional Reach Test; MFRT}

A previous study showed that the activities of dosiflexors and plantaflexors were improved when the modified functional reach test was performed [24]. When the arm reaches forward with the hip, flexion shifts the center of weight, improving activation of the tibilalis anterior and calves $[25,26]$. Because of the associated decreased muscle strength, stroke patients fall more easily than healthy adults $[27,28]$. Studies related to falls have reported that there were correlations between falls in stroke patients and assessment tools such as FRT and TUG [29]. Therefore, the modified reach test was used because it considered the muscles' functional activities associated with range of motion while reaching one's arm and prevented falling. For the test, the patient sat on a chair in a relaxed position while flexing the hip joint and the knee joint at 90 degrees and keeping the distance from the popliteal region of the posterior legs at about $5 \mathrm{~cm}$ with the feet touching the ground [30]. For anterior measurement, the shoulder joint was flexed at 90 degrees and the elbow was maximally extended to align with the wrist. At this time, the distance from the end of the fingers (middle finger) was measured. All evaluations were performed three times and the mean values were recorded [31]. The reliability of the modified reach test was high between the test $(r=.92)$ and retest $(\mathrm{r}=.92)[32]$.

\section{Statistical analysis}

SPSS version 24.0 (IBM, Korea) was used for the analyses performed in this study and the KolmogorovSmirnov test was employed to evaluate normality. The changes between pre and post intervention within the group were analyzed using the paired t-test, while those between groups were analyzed using the independent sample t-test. The statistical significance level (a) of all data was set to .05 . 
Table 1. Characteristics of subjects

\begin{tabular}{cccc}
\hline & FG $(\mathrm{n}=13)$ & FMG $(\mathrm{n}=13)$ & $\mathrm{P}$ \\
\hline Sex (Male/Female) & $7 / 6$ & $8 / 5$ & .704 \\
Stroke type (Inf./Hemo.) & $10 / 3$ & $8 / 5$ & .411 \\
Onset (Months) & $13.072 \pm 5.831$ & $14.07 \pm 5.371$ & .752 \\
Hemi-side (Lt./Rt.) & $7 / 6$ & $6 / 7$ & .714 \\
Age (years) & $74.532 \pm 12.891$ & $69.534 \pm 7.762$ & .243 \\
Height (cm) & $159.381 \pm 9.424$ & $164.533 \pm 8.442$ & .151 \\
Weight (kg) & $55.534 \pm 6.962$ & $68.923 \pm 8.351$ & .101 \\
\hline
\end{tabular}

FG: FES Group/FMG: FES Mobilization Group

Inf.: Infarction/Hemo.: Hemorrhage

Table 2. Comparison of values pre and post muscle activation of the tibialis anterior and gastrocnemius for the two groups (unit :\%RVC)

\begin{tabular}{|c|c|c|c|c|c|}
\hline & & Pre & Post & $\mathrm{t}$ & $\mathrm{P}$ \\
\hline \multirow{5}{*}{ Tibialis anterior } & FMG & $25.487 \pm 18.977$ & $37.884 \pm 17.633$ & -2.990 & $.011^{*}$ \\
\hline & FG & $22.861 \pm 15.726$ & $29.097 \pm 19.251$ & -.524 & -.600 \\
\hline & $\mathrm{t}$ & -.897 & -1.615 & & \\
\hline & $P$ & .369 & .106 & & \\
\hline & & Pre & Post & $\mathrm{t}$ & $\mathrm{P}$ \\
\hline \multirow{4}{*}{ Gastrocnemius } & FMG & $23.023 \pm 10.820$ & $26.580 \pm 12.637$ & -1.992 & $.046^{*}$ \\
\hline & FG & $30.399 \pm 12.486$ & $32.978 \pm 13.034$ & -1.646 & .126 \\
\hline & $\mathrm{t}$ & -1.974 & -1.718 & & \\
\hline & Ps & $.048 *$ & .086 & & \\
\hline
\end{tabular}

Mean \pm SD

FMG: FES Mobilization Group/FG: FES Group

*: significant difference $(\mathrm{p}<.05)$

\section{Results}

\section{General characteristics}

The characteristics of the subjects are summarized in Table 1.

2. Comparison of muscle activation of the tibialis anterior and gastrocnemius between pre and post values for the two groups

Analysis of activation of the ankle joint muscle revealed there was a significant difference between pre and post intervention in the group that received joint mobilization $(\mathrm{p}<.05) \quad($ Mean \pm SD Table 2)

3. Comparison of pre and post range of motion of dorsiflexion and plantar flexion between groups

Analysis of range of motion of the ankle joint revealed a significant difference in the active range of dorsiflexion pre and post intervention in the group that received FES 
Table 3. Comparison of range of motion of dorsiflexion and plantar flexion between pre and post treatment for the two groups (unit : degree)

\begin{tabular}{|c|c|c|c|c|c|}
\hline & & Pre & Post & $\mathrm{t}$ & $\mathrm{P}$ \\
\hline \multirow{5}{*}{ PDF } & FMG & $10.538 \pm 7.160$ & $11.807 \pm 6.706$ & -3.034 & $.010^{*}$ \\
\hline & FG & $9.923 \pm 4.172$ & $10.307 \pm 4.497$ & -1.806 & .096 \\
\hline & $\mathrm{t}$ & -.268 & -.670 & & \\
\hline & $\mathrm{P}$ & .791 & .509 & & \\
\hline & & Pre & Post & $\mathrm{t}$ & $\mathrm{P}$ \\
\hline \multirow{5}{*}{ PPF } & FMG & $17.230 \pm 13.989$ & $18.761 \pm 13.428$ & -3.596 & $.004^{*}$ \\
\hline & FG & $18.115 \pm 11.768$ & $18.453 \pm 11.972$ & -2.195 & $.049^{*}$ \\
\hline & $\mathrm{t}$ & .174 & -.062 & & \\
\hline & $\mathrm{P}$ & .863 & .951 & & \\
\hline & & Pre & Post & $\mathrm{t}$ & $\mathrm{P}$ \\
\hline \multirow{5}{*}{$\mathrm{ADF}$} & FMG & $8.307 \pm 7.398$ & $10.53 \pm 7.16$ & -2.370 & $.001^{*}$ \\
\hline & FG & $5.076 \pm 4.348$ & $5.923 \pm 5.090$ & -2.668 & $.020^{*}$ \\
\hline & $\mathrm{t}$ & -.33 & -.72 & & \\
\hline & $\mathrm{P}$ & .73 & .46 & & \\
\hline & & Pre & Post & $\mathrm{t}$ & $\mathrm{P}$ \\
\hline \multirow{4}{*}{ APF } & FMG & $11.000 \pm 12.851$ & $12.615 \pm 14.168$ & -3.148 & $.008^{*}$ \\
\hline & FG & $8.000 \pm 8.436$ & $9.230 \pm 9.696$ & -2.551 & $.025^{*}$ \\
\hline & $\mathrm{t}$ & -.298 & -.711 & & \\
\hline & $\mathrm{P}$ & .768 & .484 & & \\
\hline
\end{tabular}

Unit: degree

Mean \pm SD

PDF: Passive Dorsiflexion/PPF: Passive Plantar Flexion

ADF: Active Dorsiflexion/APF: Active Plantar Flexion

FMG: FES Mobilization Group/FG: FES Group

*: significant difference $(\mathrm{p}<.05)$

alone. There were also significant differences in active and passive ranges pre and post intervention in the group that received joint mobilization $(\mathrm{p}<.05)$ (Table 3$)$.

\section{Comparison of pre and post Modified Functional} Reach Test scores for the two groups

Comparison of the modified functional reach test scores revealed significant differences between pre and post intervention in the group that received FES and joint mobilization in combination $(\mathrm{p}<.05)$ (Table 4).

\section{Discussion}

Patients with stroke due to damage in the central nervous system suffer from muscle weakness, abnormal muscle tone, and postural control disability, resulting in difficulty with motor control [33]. Of the phenomena related to abnormal muscle tone, spasticity appears commonly in the extensor of the affected lower limb and in the flexor of the upper limb, and this is often accompanied by joint contracture, which limits the range of motion and interferes 
Table 4. Comparison of pre and post Modified Functional Reach Test results between groups

(unit: $\mathrm{cm}$ )

\begin{tabular}{cccccc}
\hline & & Pre & Post & t & P \\
\hline \multirow{3}{*}{ MFRT } & FMG & $16.112 \pm 6.382$ & $17.502 \pm 7.647$ & -2.796 & $.027^{*}$ \\
& FG & $17.194 \pm 7.691$ & $17.813 \pm 8.194$ & -.859 & .419 \\
& $\mathrm{t}$ & .304 & .079 & & \\
\hline
\end{tabular}

Unit: $\mathrm{cm}$

Mean \pm SD

MFRT: Modified Functional Reach Test

FMG: FES Mobilization Group/FG: FES Group

*: significant difference $(\mathrm{p}<.05)$

with rehabilitation [34]. Muscle activation and ROM of ankle joints are especially important in intervention programs of stroke patients. Therefore, this study investigated the effects of combined joint mobilization and FES on muscle activation and mobility of ankle joints and MFRT in stroke patients.

In clinical practice, FES is commonly used as an intervention for ankle joints in stroke patients. In a study conducted by Cho [20], FES and exercise therapy were applied together in 60 stroke patients, with FES applied to the tibialis anterior, and isometric contraction of gastrocnemius and contraction of the tibialis anterior manually applied as an exercise therapy. The results of this study showed that ROM increased gradually with decreased spasticity of the plantar flexor in the group that received both FES and exercise therapy. The changes in ROM over time were found to be statistically significant $(\mathrm{p}<.05)$, with a value of $14.64^{\circ}$ observed one week after the treatment, $7.21^{\circ}$ at week $2,4.35^{\circ}$ at week 3 , and $14.45^{\circ}$ at week $4(\mathrm{p}<.05)$. Sabut et al. [6] applied electrical stimulation to the peroneal nerve and tibialis anterior of the affected side for 20-30 minutes in combination with a traditional rehabilitation program in 51 stroke patients. They found that the muscle strength of the dorsiflexor significantly increased in the FES group and the control group by $56.6 \%$ and $27.7 \%$, respectively. Jung [17] applied muscle-strengthening exercise and FES to the tibialis anterior in 20 stroke patients and found that the dorsiflexion range of the affected side was significantly improved, even after the muscle-strengthening exercise in parallel with the FES. In the present study, the effects of combined FES and joint mobilization on muscle activity, ROM, and MFRT score were investigated by dividing the subjects into a group receiving FES only and a group receiving both FES and joint mobilization in combination. In previous studies, FES was applied only to the dorsiflexors, but in this study FES was also applied to the plantar flexors. No significant differences in the measurements of muscle activity were observed in the group that received FES alone, but the magnitude of the mean values pre and post intervention increased. The mean value of the passive range of motion (PROM) increased, while that of the active range of motion (AROM) increased significantly $(\mathrm{p}<.05)$. The method of antagonist stimulation was used to facilitate the dorsiflexors induced ankle dorsiflexion and inhibition of plantaflexion spasticity during the stance phase, the tone of plantaflexors was decreased and ROM was increased [16]. Thus, in this study the antagonist stimulation method also showed a positive effect as same results.

In contrast, in a study conducted by Hur [35], AROM increased after treatment in the group that received combined FES and active extension, but no significant difference was found in the group that received FES alone $(\mathrm{p}<.05)$. At this time, the frequency of stimulation was 
five times a week for 20 minutes per session. In the present study, the frequency of stimulation was four times a week for 30 minutes per session. Considering that a significant difference was observed in the electrical stimulation level at 30 minutes [36], the method applied in the present study seemed to positively affect AROM. Therefore, these results suggest that the FES-mediated intervention helped the subject recognize each joint and muscle activation through neurological feedback [37], thereby exerting a positive effect on the average values of muscle activation and ROM, even in the group that received FES alone.

In addition to FES therapy, there is a need to improve passive and active movement by reinforcing muscular strength around the ankle as an intervention for stroke patients [8]. Kaltenborn [19] reported that in joint mobilization, passive joint play increased following the application of passive motion, which led to increased active movement. In a study conducted by Lee et al. [10], 34 stroke patients were divided into two groups. In the experimental group, posterior talus glide was applied in conjunction with dorsiflexion, while the placebo group was instructed to perform dorsiflexion exercise slowly without lifting the heel from the sofa until no pain was felt. As a result, the PROM of the dorsiflexor was significantly improved in the group that received joint mobilization. An and Won [8] applied Mobilization with Movement (MWM), which is a Mulligan therapy, to the talocrural joints of 38 stroke patients.

The muscle strength of the dorsiflexor of the ankle joint reached the maximum torque value after intervention in the MWM group. The same result appears to have been obtained because the shin of talus was made to glide forward using a belt. In a study conducted by Kim [38], 30 stroke patients were divided into an experimental group and a control group, then subjected to joint mobilization accompanied by weight bearing for four weeks. The ROM and dynamic balance ability were improved in the experimental group $(p<.05)$, suggesting that the intervention was effective. In the present study, a significant difference $(\mathrm{p}<.05)$ was observed in the muscle activity of the group that received combined FES and joint mobilization $(\mathrm{p}<.05)$. When ROM was measured, a significant difference in the ranges of both active and passive motions was also observed $(\mathrm{p}<.05)$; therefore, the application of joint mobilization increased the mobility of the ankle joint with limited mobility. These results indicate that combined application of FES and joint mobilization have a positive effect on muscle activity and ROM, which is consistent with the results of previous studies.

As shown above, intervention on ankle joints improves structural function, thereby improving balance ability. In clinical practice, the $10 \mathrm{M}$ walking test, timed up and go test, step test, and FRT are used to evaluate the balance ability of stroke patients [39]. Among these, FRT is often used to evaluate static balance ability to determine forward stability limits [40]. Kim et al. [11] applied FES to the dorsiflexor and performed proprioceptive training and muscle strength training of ankle joints in 22 stroke patients. In the control group, ankle joint stretching was performed with the application of FES. The results revealed significant increases in the FRT score 3 and 6 weeks after the start of training in both groups compared to before training. In the control group, the FRT improved from 18.6 55.3 at the initial evaluation to $22.5 \pm 4.8$ after 3 weeks and to $24.1 \pm 4.6$ after 6 weeks. In the experimental group, a greater improvement from $18.0 \pm 7.9$ at the initial evaluation to $23.6 \pm 7.5$ after 3 weeks and $26.6 \pm 7.0$ after 6 weeks was observed. Considering that improvement of the FRT score reflects improvement of anterior/posterior movements of the ankle joint[41], these results suggest that there was a positive effect on balance ability in a sitting posture, consistent with the findings of previous studies showing that combining FES with exercise therapy is effective.

Unlike previous studies, in this study, the electrodes for FES were attached to the anterior and posterior regions of the tibialis anterior and gastrocnemius. Balance control 
for anterio-posterior shaking depends on the alternating activation of the tibialis anterior and gastrocnemius [42]. Therefore, it is believed that intervention through joint mobilization following the promotion of alternating activation by FES improved the structural function (muscle activity and mobility), which in turn enhanced the anterioposterior control ability (modified functional reach test).

It should be noted that this study was limited in that pre and post intervention effects were measured after four weeks, the follow-up effects were not examined, and the sample size was small. Therefore, in the future, systematic research should be conducted to monitor the long-term effects in a large number of stroke patients.

In conclusion, the intervention method combining FES with joint mobilization was found to be more effective at improving muscle activation, mobility, and MFRT than the application of FES alone during ankle joint rehabilitation of stroke patients.

\section{Conclusion}

This study investigated the effects of combined FES on the dorsiflexor and plantar flexor of the ankle joint and joint mobilization on muscle activation, mobility, and MFRT. The results of this study showed that there were significant improvements in muscle activation, ROM, and MFRT $(p<.05)$ in the group that received combined FES and joint mobilization compared to the group that received FES alone $(p<.05)$. Therefore, the results of this study suggest that a systematic program of proactive posture control can be used to prevent dysfunction when planning interventions for ankle joints as a way to help stroke patients walk efficiently.

\section{Acknowledgements}

This paper was supported by the research fund offered by the Catholic University of Pusan and a National
Research Foundation of Korea (NRF) grant (No. 2017R1C1B5074040) funded by the Korean government (MSIT).

\section{References}

[1] Burridge JH, Wood DE, Taylor PN, et al. Indices to describe different muscle activation patterns, identified during treadmill walking, in people with spastic drop-foot. Med Eng Phys. 2001;23(6):427-34.

[2] Harlaar J, Becher JG, Snijders CJ, et al. Passive stiffness characteristics of ankle plantar flexors in hemiplegia. Clin Biomech. 2000;15(4):261-70.

[3] Baker LL, Yeh C, Wilson D et al. Electrical stimulation of wrist and fingers for hemiplegic patients. Phys Ther. 1979;59(12):1495-9.

[4] Morone G, Fusco A, Capua PD, et al. Walking Training with Foot Drop Stimulator Controlled by a Tilt Sensor to Improve Walking Outcomes: A Randomized Controlled Pilot Study in Patients with Stroke in Subacute Phase. Stroke Res Treat. 2012;(2012):523-64.

[5] Tiebin Yan, CWY Hui-Chan, LSW Li. Functional Electrical Stimulation Improves Motor Recovery of the Lower Extremity and Walking Ability of Subjects With First Acute Stroke. Stroke. 2005;36:80-5.

[6] Sabut SK, Sikdar C, Kumar R, et al. Functional electrical stimulation of dorsiflexor muscle: Effects on dorsiflexor strength, plantarflexor spasticity, and motor recovery in stroke patients. NeuroRehabilitation. 2011;29(4):393400.

[7] Sabut SK, Bhattacharya SD, Manjunatha M. Functional electrical stimulation on improving foot drop gait in poststroke rehabilitation: a review of its technology and clinical efficacy. Crit Rev Biomed Eng. 2013;41(2): 149-60.

[8] An CM, Won J. Effects of Ankle Joint Mobilization with Movement on Lower Extremity Muscle Strength and Spatiotemporal Gait Parameters in Chronic 
Hemiplegic Patients. Phys Ther Korea. 2012;19(3):20-9.

[9] An CM, Won J. Effects of ankle joint mobilization with movement and weight-bearing exercise on knee strength, ankle range of motion, and gait velocity in patients with stroke: a pilot study. J Phys Ther Sci. 2016;28(2):689-94.

[10] Lee J, Kim JO, Lee BH .The effects of posterior talar glide with dorsiflexion of the ankle on mobility, muscle strength and balance in stroke patients: a randomised controlled trial. J Phys Ther Sci. 2017;29(3):452-56.

[11] Kim K, Lee S, Kim D, et al. The effects of ankle joint muscle strengthening and proprioceptive exercise programs accompanied by functional electrical stimulation on stroke patients' balance. J Phys Ther Sci. 2015;27(9):2971-5.

[12] Runge CF, Shupert CL, Horak FB, et al. Ankle and hip postural strategies defined by joint torques. Gait \& Posture. 1999;10(2):161-70.

[13] Park KH. The Effect of Ankle Strategy Exercises on Balance of Patients with Hemiplegia. Master's Degree Daegu University. 2013.

[14] Jeon SN. Impact of Ankle Strategy Exercise on Balancing Ability of Patients with Stroke According to the Existence of Visual Feedback. Master's Degree Namseoul University. 2015.

[15] Mun BM. Effect of Strengthening Exercise with Lower Extremity and Functional Electrical Stimulation on Gait, Balance and Spasticity in Chronic Stroke Patients. Doctor's Degree Daegu University. 2014.

[16] Kang YH, Toon SE, Seo SK, et al. The Effect on Change of Spinal Neuron Excitability during Gait Training of Hemiplegia Patients by the Functional Electrical Stimulation. J Phys Ther Sci. 2007;19(1):11-22.

[17] Jung TW. The Effects of Functional Electrical Stimulation with Progressive Resistance Exercise on Dorsiflexors of Hemiparesis Side for The Patients with Stroke. Master's Degree DanKook University. 2013.

[18] Stewart C, Postans N, Schwartz MH, et al. An exploration of the function of the triceps surae during normal gait using functional electrical stimulation. Gait \& Posture.
2007;26(4):482-8.

[19] Kaltenborn. Manual Mobilization of the Joints (7th ed). Yeongmun Publisher. Orthopedic Physical Therapy Products. 2011

[20] Cho MS. The Effect on Ankle Joint Movement by FES Application on Tibialis Anterior Muscle in Chronic Stroke Patients. J Kor Soc Med. 2011;6(3):277-86.

[21] Nam SM. The Effects on Muscle Activation and Reaction Time of The Balance Trainer Controlled Knee-joint Mobility in Functional Ankle Instability Adult Males. Master's Degree Daegu University. 2015.

[22] Moon GS. The kinematic analysis of the ankle joint and EMG analysis of the Lower Limbs muscle for the different walking speed. Sports Biomech. 2005;15(1):177-95.

[23] Park MC, Lee SY. Deltoid Middle Fiber of the Isometric Contraction According to Elastic Band and Dumbbell on Various Shoulder Abduction Angle. J Korean Soc Phys Med 2016;11(2):71-6.

[24] Dean CM, Shepherd R. Task-related training improves performance of seated reaching tasks after stroke: a randomized controlled trial. Stroke. 1997;28(4):722-8.

[25] Bock C, Gentile AM, Kaminski TR. The coordination between trunk and arm motion during pointing movements. Exp Brain Res. 1995;106(3):457-66.

[26] Mastalerz A, Palczewska IW. The influence of trunk inclination on muscle activity during sitting on forward inclined seats. Acta Bioeng Biomech. 2010;12(4):19-24

[27] Rapport, LJ, Websster JS, Flemming KL, et al. Predictors of fall among right-hemisphere stroke patients in the rehabilitation setting. Arch Phys Med Rehabil. 1993; 74(6):621-6.

[28] Mackintosh SF, Hill KD, Dodd KJ, et al. Balance score and a history of falls in hospital predict recurrent falls in the 6 months following stroke rehabilitation. Arch Phys Med Rehabil. 2006;87(12):1583-9.

[29] Lee HS, Choi JH. Correlation between BBS, FRT, STI, TUG, MBI, and falling in stroke patients. The Journal of Korean Society of Physical Therapy. 2008;20(4):1-6. 
[30] Lynch SM, Leahy P, Barker SP. Reliability of measurements obtained with a modified functional reach test in subjects with spinal cord injury. Phys Ther. 1998;78(2):128-33.

[31] Katz-Leurer M, Rotem H, Keren O, et al. Balance abilities and gait characteristics in post-traumatic brain injury, cerebral palsy and typically developed children. Dev Neurorehabil. 2009;12(2):100-5.

[32] Duncan PW, Weiner DK, Chandler J, et al. Functional reach: a new clinical measure of balance. J Gerontol. 1990;45(6):192-7.

[33] Carr JH, Shepherd RB, Nordholm L, et al. Investigation of a new motor assessment scale for stroke patient. Phys Ther. 1985;65(2):175-80.

[34] Welmer AK, Holmqvist LW, Disa K, et al. Hemiplegic limb synergies in stroke patients. Am J Phys Med Rehabil. 2006;85(2):112-9.

[35] Hur YG. The Effects on Spasticity, Range of Motion, Balance on Combining Active-Stretching Exercise of Plantarflexor on Ankle with FES in The Patients with Stroke. Master's Degree Yongin University. 2013.
[36] Jin HK, Cho SH. The Effect of Electrical Stimulation Level on Autonomic Nerve System in Normal Adults. Journal of the Korea Academia-Industrial cooperation Society. 2015;16(3):2029-37.

[37] Sheffler LR, Chae J. Neuromuscular electrical stimulation in neurorehabilitation. Muscle Nerve. 2007;35(5):562-90.

[38] Kim SL. The Effects of Weight Bearing based Mobilization with Movement on Balance and Gait in Stroke Patients. Master's Degree SahmYook University. 2015.

[39] Bower KJ, McGinley JL, Miller KJ, et al. Instrumented Static and Dynamic Balance Assessment after Stroke Using Wii Balance Boards: Reliability and Association with Clinical Tests. PLoS One. 2014;9(12):115-282.

[40] Jeong SB. The Effects of Lower Extremity Muscle Strength Exercise of 8 Weeks on the Balance and Gait in Stroke Patients. Master's Degree Yongin University. 2013.

[41] Winter DA, Paltla AE, Prince F. Stiffness control of balance in quiet standing. J Neurophysiol. 1998;80(3): 1121-211.

[42] Almeida GL, Carvalho RL, Talis VL. Postural strategy to keep balance on the seesaw. Gait \& posture. 2006; 23(1):17-21. 